\title{
Cerebral blood flow in children with syndromic craniosynostosis: cohort arterial spin labeling studies
}

\author{
Priya N. Doerga, MD, ${ }^{1}$ Maarten H. Lequin, MD, PhD, ${ }^{2}$ Marjolein H. G. Dremmen, MD, ${ }^{3}$ \\ Bianca K. den Ottelander, MD, ${ }^{1}$ Katya A. L. Mauff, ${ }^{4}$ Matthias W. Wagner, MD, ${ }^{5-7}$ \\ Juan A. Hernandez-Tamames, PhD, ${ }^{3}$ Sarah L. Versnel, MD, PhD, ${ }^{1}$ \\ Koen F. M. Joosten, MD, PhD, ${ }^{8}$ Marie-Lise C. van Veelen, MD, PhD, ${ }^{9}$ \\ Robert C. Tasker, MA, MD, FRCP, ${ }^{10}$ and Irene M. J. Mathijssen, MD, PhD, MBA-H ${ }^{1}$
}

\begin{abstract}
1'Department of Plastic and Reconstructive Surgery and Hand Surgery, Dutch Craniofacial Center, ${ }^{3}$ Department of Radiology, ${ }^{4}$ Department of Biostatistics, ${ }^{8}$ Pediatric Intensive Care Unit, and ' Department of Neurosurgery, Sophia Children's Hospital, Erasmus MC, University Medical Center Rotterdam; '2Department of Radiology, University Medical Center Utrecht, The Netherlands; ${ }^{5}$ Department of Radiology and Radiological Science, Section of Pediatric Neuroradiology, Division of Pediatric Radiology, The Johns Hopkins University School of Medicine, Baltimore, Maryland; ${ }^{6}$ Institute of Diagnostic and Interventional Radiology, University Hospital Zurich, University of Zurich, Switzerland; ${ }^{7}$ Department of Diagnostic Imaging, Division of Neuroradiology, The Hospital for Sick Children, Toronto, ON, Canada; and ${ }^{10}$ Departments of Neurology and Anesthesiology (Pediatrics), Harvard Medical School and Boston Children's Hospital, Boston, Massachusetts
\end{abstract}

\begin{abstract}
OBJECTIVE In comparison with the general population, children with syndromic craniosynostosis (sCS) have abnormal cerebral venous anatomy and are more likely to develop intracranial hypertension. To date, little is known about the postnatal development change in cerebral blood flow (CBF) in SCS. The aim of this study was to determine CBF in patients with SCS, and compare findings with control subjects.
\end{abstract}

METHODS A prospective cohort study of patients with SCS using MRI and arterial spin labeling (ASL) determined regional CBF patterns in comparison with a convenience sample of control subjects with identical MRI/ASL assessments in whom the imaging showed no cerebral/neurological pathology. Patients with SCS and control subjects were stratified into four age categories and compared using CBF measurements from four brain lobes, the cerebellum, supratentorial cortex, and white matter. In a subgroup of patients with SCS the authors also compared longitudinal pre- to postoperative CBF changes.

RESULTS Seventy-six patients with sCS ( 35 female [46.1\%] and 41 male [53.9\%]), with a mean age of 4.5 years (range 0.2-19.2 years), were compared with 86 control subjects (38 female [44.2\%] and 48 male [55.8\%]), with a mean age of 6.4 years (range $0.1-17.8$ years). Untreated SCS patients $<1$ year old had lower CBF than control subjects. In older age categories, $\mathrm{CBF}$ normalized to values observed in controls. Graphical analyses of $\mathrm{CBF}$ by age showed that the normally expected peak in CBF during childhood, noted at 4 years of age in control subjects, occurred at 5-6 years of age in patients with sCS. Patients with longitudinal pre- to postoperative CBF measurements showed significant increases in CBF after surgery. CONCLUSIONS Untreated patients with $\mathrm{SCS}<1$ year old have lower CBF than control subjects. Following vault expansion, and with age, $\mathrm{CBF}$ in these patients normalizes to that of control subjects, but the usual physiological peak in CBF in childhood occurs later than expected.

https://thejns.org/doi/abs/10.3171/2019.10.PEDS19150

KEYWORDS syndromic craniosynostosis; controls; childhood; cerebral blood flow; preoperative; postoperative; craniofacial

$\mathrm{S}$ YNDROMIC craniosynostosis (sCS) is a congenital disorder in which several skull sutures close prematurely, causing facial, calvarial, and skull base anomalies. ${ }^{15}$ This disorder occurs in 0.9 per 10,000 live births, ${ }^{7}$ and is associated with additional congenital anom- alies and intracranial hypertension (ICH). ${ }^{17,21}$ In these children ICH is mainly seen in the first 6 years of life, and can ultimately lead to vision loss. ${ }^{5}$ Hence, many craniofacial surgical treatment centers recommend a cranial vault expansion before 1 year of age for all children with $\mathrm{sCS}$

ABBREVIATIONS ANCOVA = analysis of covariance; $A S L$ = arterial spin labeling; $C B F=$ cerebral blood flow; $I C H$ = intracranial hypertension; ICP = intracranial pressure; MANCOVA = multivariate ANCOVA; PLD = postlabeling delay; ROI = region of interest; $\mathrm{SCS}$ = syndromic craniosynostosis; VP = ventriculoperitoneal.

SUBMITTED March 18, 2019. ACCEPTED October 21, 2019.

INCLUDE WHEN CITING Published online December 27, 2019; DOI: 10.3171/2019.10.PEDS19150. 
to prevent $\mathrm{ICH} .3,18,20$ The reasoning here is that physical constraint such as delayed skull growth and progressive expansion of CSF ventricles are important factors in the development of ICH. However, it is also possible that the problem is physiological and related to alteration in cerebral blood flow (CBF) development and dynamics.

To date, only limited research has been conducted on characterizing the developmental progression of CBF in children with sCS, in part because the technical needs for assessment have been invasive or required using radiation, contrast media, and nuclear medicine..$^{2,16,26}$ Such research focused on how vault expansion affects CBF, with no comparison to data from control subjects. ${ }^{8,23,24}$ Now, newer and validated techniques for CBF assessment using MRI with arterial spin labeling (ASL) mean that measurements can be made without using contrast agents and radiation exposure. Therefore, in this study we aimed to determine $\mathrm{CBF}$ in patients with sCS, and compare the findings to normal values from healthy children $0-18$ years old. Our research questions were: 1) does $\mathrm{CBF}$ in untreated patients with sCS differ from that of control subjects; 2) does CBF in patients with sCS following vault expansion differ from that of control subjects; and 3) does CBF improve in sCS patients with serial CBF measurements pre- to postsurgical vault expansion?

\section{Methods}

The Ethics Committee of the Erasmus Medical Center approved this prospective imaging study in patients with sCS. In our national sCS center (Erasmus Medical Center), all patients are managed according to a clinical protocol that since 2012 has included MRI with an ASL sequence, after referral (usually $<1$ year of age) and when 4 years old. Patients with Crouzon syndrome undergo additional MRI when they are 2 years of age because of their high risk of developing Chiari malformation type I. Patients with sCS were included as a consecutive sample starting from 2012. Children $<7$ years old generally undergo deep sedation or anesthesia during the MRI procedure, which includes using propofol and sevoflurane.

The data used to represent control information for sCS comparisons were generated as a convenience sample ${ }^{25}$ from subjects undergoing MRI for clinical reasons, but the following conditions were met: 1) the subjects were found to have no neurological pathology of the head and neck area; 2) the subjects were free of any neurological or psychological morbidity on follow-up; and 3) the subjects' MRI data were of sufficient quality to be used for research. Indications for MRI with ASL sequence in these control subjects were to follow up on extracranial pathology such as rhabdomyosarcoma, epithelioma, and facial port-wine stain, and to exclude intracranial pathology in cases of abnormal headaches, behavioral disorders, and brief minor neurological deficit without sequelae.

\section{MRI Data}

MRI was performed on a 1.5-T unit (General Electric Healthcare), including pseudocontinuous ASL sequences, with the following imaging parameters: TR $4604 \mathrm{msec}$, TE $10.7 \mathrm{msec}$, voxel size $3.75 \times 3.75 \times 4.0 \mathrm{~mm}$, field of view 24.0, postlabeling delay (PLD) for children $<2$ years $1025 \mathrm{msec}$, PLD for children > 2 years $1500 \mathrm{msec}$. Data processing was performed using the software program Advantage Workstation Server (General Electric Healthcare). Quantitative perfusion maps were generated based on a tissue relaxation time of 1.5 seconds and lambda of 0.9. Equilibrium magnetization of blood was estimated from the data.

ASL images were coregistered with T2- and T1-weighted MR images. Measurement of CBF was performed by manually placing regions of interest (ROIs) on T2- and T1weighted images, after which the corresponding location and $\mathrm{CBF}$ value on the ASL image were extrapolated. We manually placed ROIs, because automated programs perform ROI placement with lower accuracy in patients with sCS, because of their distorted brain anatomy. ${ }^{22} \mathrm{~A}$ measurement protocol based solely on anatomical landmarks was constructed with 47 ROIs distributed over frontal, parietal, temporal, and occipital lobes, and the cerebellum. Each ROI was placed on three consecutive slices in which the structure of interest was visible. We then averaged the three values. If a structure was not visible on all three slices, the average was calculated using slices on which it was visible. The evaluator was not blinded to whether a subject was a control subject or sCS patient. True blinding of the evaluator was impossible, because the abnormal head shape is visible on MR images. However, the evaluator closely followed the measurement protocol based solely on anatomical landmarks, minimizing the need for individual interpretations, and thereby minimizing the potential bias of not blinding the evaluator.

\section{Statistical Analyses}

Statistical analyses were performed using IBM SPSS statistical program (version 24, IBM Corp.) and R software ( $\mathrm{R}$ Foundation for Statistical Computing). To determine the reproducibility of the measurement protocol, average-measures 2-way mixed intraclass correlation coefficients tested intra- and interrater reliability. P.N.D. performed all ASL measurements. Intrarater reliability was tested (P.N.D.) by repeating ASL measurements in a subset of 10 control subjects. Interrater reliability was tested (B.K.D.O.) by performing ASL measurements in a subset of 10 control subjects.

\section{Cross-Sectional Measurements: sCS Patients Compared to Controls}

Differences in CBF of cross-sectional measurements between untreated patients, treated patients, and control subjects were examined in supratentorial and infratentorial brain areas, including data from the frontal, parietal, occipital, and temporal lobes, and cerebellum. In supratentorial areas, cortex and white matter were separately assessed. Multivariate analysis of covariance (MANCOVA) determined possible differences as a multiple model and, as such, determined whether or not factors (such as patients vs controls) influenced the relationship between responses (such as CBF of brain lobes and cerebellum). Analyses of covariance (ANCOVAs) determined possible differences as univariate models and, as such, assessed the 
TABLE 1. Characteristics in patients with sCS and controls

\begin{tabular}{|c|c|c|c|c|}
\hline & \multicolumn{3}{|c|}{ sCS Pts } & \multirow[b]{2}{*}{ Controls, $n=86$} \\
\hline & Total, $n=76$ & Untreated, $n=36$ & Treated, $n=40$ & \\
\hline Mean age (range), yrs & $4.5(0.20-19.18)$ & $1.5(0.20-10.67)$ & $7.24(1.15-19.18)$ & $6.37(0.1-17.78)$ \\
\hline Females, n (\%) & $35(46.1)$ & $18(50)$ & $17(42.5)$ & $38(44.2)$ \\
\hline Males, $\mathrm{n}(\%)$ & $41(53.9)$ & $18(50)$ & $23(57.5)$ & $48(55.8)$ \\
\hline \multicolumn{5}{|l|}{ Syndrome, n (\%) } \\
\hline Apert & $14(18.4)$ & 4 & 10 & \\
\hline Crouzon & $23(30.3)$ & 11 & 12 & \\
\hline Muenke & $8(10.5)$ & 2 & 6 & \\
\hline Saethre-Chotzen & $3(3.9)$ & 3 & 0 & \\
\hline Complex & $18(23.7)$ & 8 & 10 & \\
\hline Carpenter & $2(2.6)$ & 2 & 0 & \\
\hline Unicoronal synostosis & $6(7.9)$ & 4 & 2 & \\
\hline Unknown craniosynostosis syndrome & $2(2.6)$ & 2 & 0 & \\
\hline \multicolumn{5}{|l|}{ Age categories } \\
\hline I (<1 yr) & 27 & 27 & 0 & 8 \\
\hline II (1 to <3 yrs) & 7 & 4 & 3 & 17 \\
\hline III (3 to <8 yrs) & 29 & 4 & 25 & 34 \\
\hline IV ( $\geq 8$ yrs) & 13 & 1 & 12 & 27 \\
\hline
\end{tabular}

influence of factors on a single response at a time. Variables that we considered for the MANCOVA model were age, sex, and sedation technique (sevoflurane, propofol, combination of sevoflurane and propofol, or awake). We first ran a full model with all covariates for each age category and included covariates that had a $p$ value $<0.10$ in the final model. Only age had $\mathrm{p}$ values below 0.10 .

We stratified age into four categories as used in previous studies: ${ }^{28}$ category I ( 0 to $<1$ year old $)$, category II ( 1 to $<3$ years old), category III ( 3 to $<8$ years old), and category IV ( 8 years and older). In these categories, we were able to make the following comparisons: category I, comparing untreated patients to controls; category II, comparing untreated and treated patients to controls; and categories III and IV, comparing treated patients to control subjects. The $p$ values of MANCOVAs and ANCOVAs within age categories were adjusted for multiple testing with a Bonferroni correction. The level of significance was set at 0.05 . The $p$ values were not further adjusted between age categories. There were two reasons we chose age stratification. The first reason was to facilitate comparisons to other literature on the subject. The second was that a regression model, while more sensitive, could have introduced bias by grouping all sCS patients together, because younger patients were more often untreated and older patients were more often treated.

Age-related progression of CBF for controls, untreated, and treated patients was graphically assessed. Predicted mean $\mathrm{CBF}$ profiles for each group were obtained via linear regression using $\mathrm{B}$ splines of age. These mean profiles, together with $95 \%$ confidence interval (CI) bands for the control group, were then used to visualize further differences in the progression of CBF patterns over time between the controls and the treated and untreated patients.

\section{Longitudinal Measurements}

Age-related progression of $\mathrm{CBF}$ in patients with longitudinal pre- to postoperative measurements was graphically assessed. Patient-specific line graphs for individuals with longitudinal data were compared to the mean $\mathrm{CBF}$ profile and $95 \%$ CI bands for controls to determine differences in age-related progression of $\mathrm{CBF}$. The $\mathrm{McNe}-$ mar chi-square test determined longitudinal differences in proportions of patients below 95\% CI bands pre- and postoperatively.

\section{Results}

Table 1 summarizes the demographic data of 76 patients with sCS and 86 control subjects. This study included patients with Apert, Crouzon, Muenke, and Saethre-Chotzen syndromes. Complex craniosynostosis refers to patients in whom the genetic cause is unknown but expected because multiple sutures are involved. Nine patients had hydrocephalus, 1 patient had a ventriculoperitoneal (VP) drain, and 7 patients had papilledema. Exploratory analysis showed that CBF data of these patients were within \pm 3 standard deviations (SDs) of the estimated mean of sCS patients' brain regions and brain structures, and hence were not outliers (Table 2).

Intrarater reliability tests for ROI ASL measurements resulted in an intraclass correlation coefficient of 0.99 (95\% CI 0.93-0.99). Interrater reliability tests for ROI ASL measurements resulted in an interclass correlation coefficient of 0.91 (95\% CI 0.57-0.98).

\section{Patients With sCS Compared to Controls}

Overall, we found no significant differences in CBF be- 


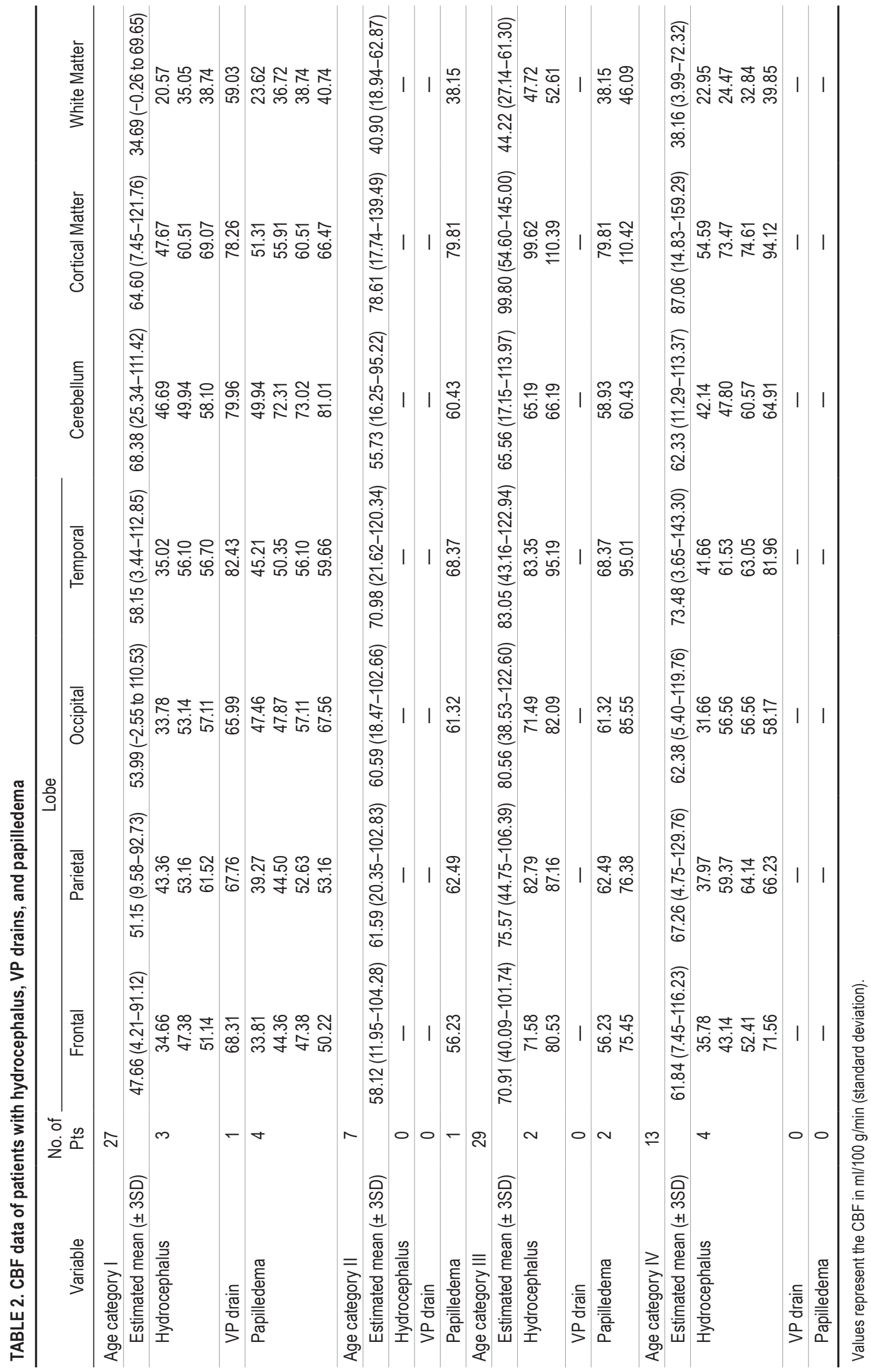


TABLE 3. Mean CBF in controls and sCS patients in age categories I-IV

\begin{tabular}{|c|c|c|c|c|c|c|}
\hline \multirow[b]{2}{*}{ Age Category } & \multicolumn{2}{|c|}{ Controls } & \multicolumn{2}{|c|}{ Pts } & \multirow[b]{2}{*}{ ANCOVA $p$ Value } & \multirow[b]{2}{*}{ MANCOVA p Value } \\
\hline & Mean & SE & Mean & SE & & \\
\hline \multicolumn{7}{|l|}{ Category I (<1 yrs)* } \\
\hline Supratentorial \& infratentorial areas & & & & & & $<0.004$ \\
\hline Frontal lobe & 67.338 & 4.429 & 47.663 & 2.409 & $<0.001$ & \\
\hline Parietal lobe & 66.090 & 4.466 & 51.154 & 2.430 & 0.006 & \\
\hline Occipital lobe & 67.606 & 5.196 & 53.992 & 2.827 & 0.028 & \\
\hline Temporal lobe & 74.635 & 5.318 & 58.146 & 2.893 & 0.010 & \\
\hline Cerebellum & 75.519 & 5.906 & 68.380 & 3.213 & 0.296 & \\
\hline Supratentorial: cortex \& white matter & & & & & & 0.006 \\
\hline Cortex & 83.578 & 5.317 & 64.603 & 2.892 & 0.004 & \\
\hline White matter & 48.857 & 3.634 & 34.691 & 1.977 & 0.002 & \\
\hline \multicolumn{7}{|l|}{ Category II (1 to <3 yrs) } \\
\hline Supratentorial \& infratentorial areas & & & & & & 0.097 \\
\hline Frontal lobe & 76.629 & 4.108 & 58.117 & 6.403 & 0.024 & \\
\hline Parietal lobe & 76.171 & 4.053 & 61.590 & 6.317 & 0.066 & \\
\hline Occipital lobe & 82.319 & 4.052 & 60.563 & 6.315 & 0.009 & \\
\hline Temporal lobe & 88.620 & 4.300 & 70.977 & 6.702 & 0.038 & \\
\hline Cerebellum & 75.811 & 6.773 & 55.734 & 10.555 & 0.124 & \\
\hline Supratentorial: cortex \& white matter & & & & & & 0.067 \\
\hline Cortex & 99.858 & 4.723 & 78.614 & 7.361 & 0.024 & \\
\hline White matter & 54.232 & 2.857 & 40.903 & 4.453 & 0.020 & \\
\hline \multicolumn{7}{|l|}{ Category III ( 3 to $<8$ yrs) } \\
\hline Supratentorial \& infratentorial areas & & & & & & 0.020 \\
\hline Frontal lobe & 77.427 & 2.205 & 70.913 & 2.574 & 0.060 & \\
\hline Parietal lobe & 75.559 & 2.290 & 75.570 & 2.674 & 0.998 & \\
\hline Occipital lobe & 76.234 & 2.809 & 80.561 & 3.279 & 0.322 & \\
\hline Temporal lobe & 84.247 & 2.592 & 83.048 & 3.025 & 0.765 & \\
\hline Cerebellum & 64.229 & 2.419 & 65.558 & 2.823 & 0.723 & \\
\hline Supratentorial: cortex \& white matter & & & & & & 0.005 \\
\hline Cortex & 100.060 & 2.916 & 99.800 & 3.404 & 0.954 & \\
\hline White matter & 49.072 & 1.351 & 44.221 & 1.577 & 0.023 & \\
\hline \multicolumn{7}{|l|}{ Category IV ( $\geq 8$ yrs) } \\
\hline Supratentorial \& infratentorial areas & & & & & & 0.002 \\
\hline Frontal lobe & 68.952 & 2.269 & 61.842 & 3.404 & 0.091 & \\
\hline Parietal lobe & 66.389 & 2.668 & 67.256 & 4.003 & 0.858 & \\
\hline Occipital lobe & 65.813 & 2.690 & 62.378 & 4.035 & 0.483 & \\
\hline Temporal lobe & 72.875 & 2.974 & 73.478 & 4.461 & 0.911 & \\
\hline Cerebellum & 56.977 & 2.295 & 62.329 & 3.443 & 0.204 & \\
\hline Supratentorial: cortex \& white matter & & & & & & 0.066 \\
\hline Cortex & 89.650 & 3.126 & 87.059 & 4.689 & 0.648 & \\
\hline White matter & 42.802 & 1.532 & 38.155 & 2.297 & 0.101 & \\
\hline
\end{tabular}

Values represent the $\mathrm{CBF}$ in $\mathrm{ml} / 100 \mathrm{~g} / \mathrm{min}$. Boldface type indicates statistical significance.

*Age category I = untreated patients; age category II = untreated and treated patients; age categories III and IV = treated patients.

tween males and females in their white matter (controls: mean difference $0.76 \mathrm{ml} / 100 \mathrm{~g} / \mathrm{min}, 95 \% \mathrm{CI}-5.30$ to 3.78 , $\mathrm{p}=0.74$; sCS patients: mean difference $1.42 \mathrm{ml} / 100 \mathrm{~g} / \mathrm{min}$, $95 \% \mathrm{CI}-6.06$ to $3.22, \mathrm{p}=0.54$ ) or cortical matter (controls: mean difference $4.03 \mathrm{ml} / 100 \mathrm{~g} / \mathrm{min}, 95 \% \mathrm{CI}-3.97$ to 12.04, $\mathrm{p}=0.32$; sCS patients: mean difference $1.27 \mathrm{ml} / 100$ $\mathrm{g} / \mathrm{min}, 95 \% \mathrm{CI}-12.20$ to $9.66, \mathrm{p}=0.82)$. Hence, in the following presentation of results the data by sex have been grouped.

Table 3 summarizes the CBF differences between control subjects and sCS patients in age categories I-IV. In age category I, by univariate analysis (ANCOVA), CBF is 

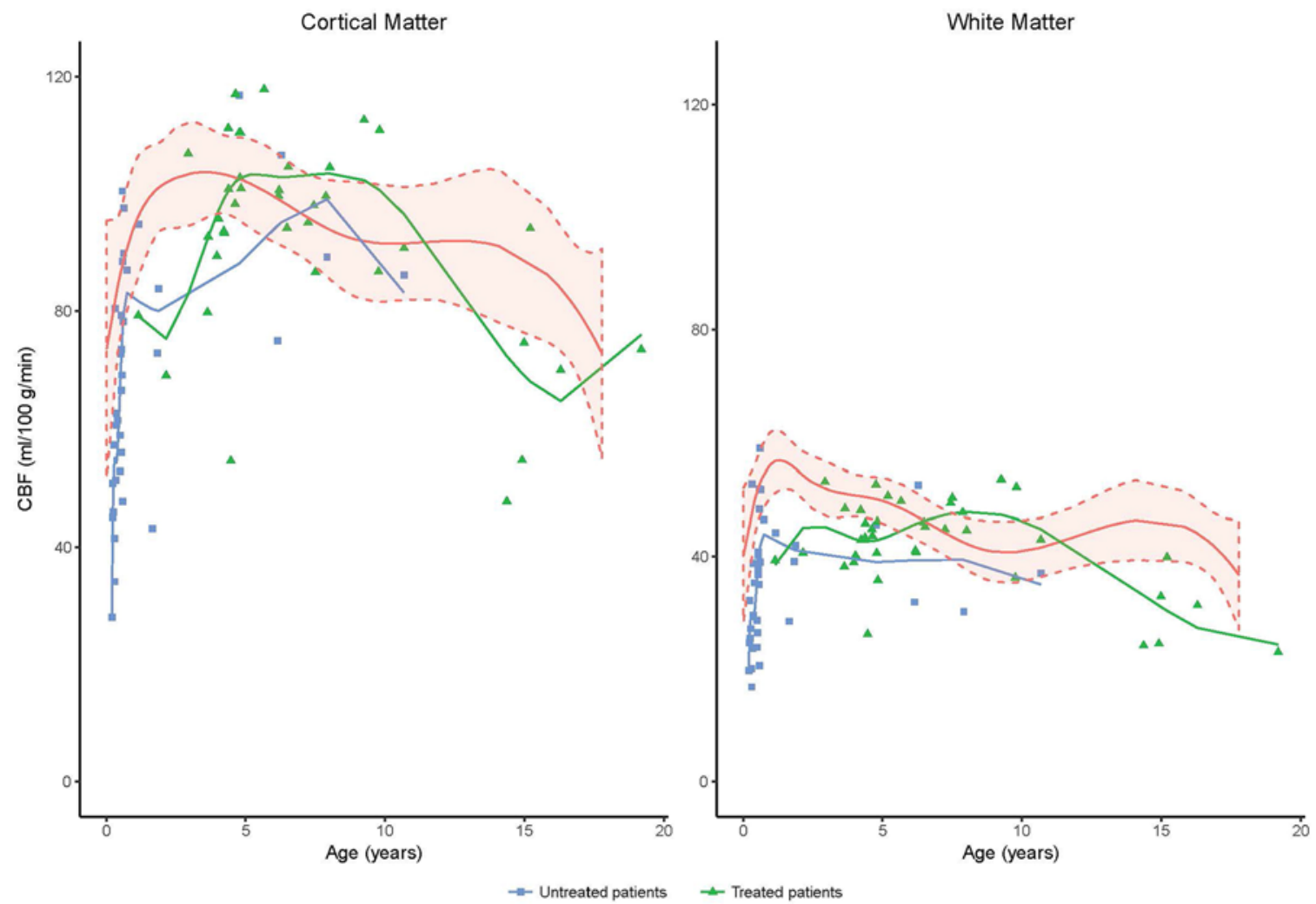

FIG. 1. Distribution of CBF for the supratentorial cortex and white matter in untreated and treated sCS patients by age at measurement, plotted against the distribution of control subject CBF data by age (mean and $95 \% \mathrm{Cl}$ represented by red line and pink shading, respectively). Figure is available in color online only.

significantly lower in untreated sCS patients for all brain lobes, cortical matter, and white matter. On multivariate analysis (MANCOVA) of supratentorial and infratentorial areas, as well as supratentorial cortex and white matter, significant differences remained between untreated sCS patients and controls. In age category II, multivariate analyses failed to identify any significant differences. In age categories III and IV these absolute CBF differences between sCS patients and controls are smaller. The apparent discrepancy in age categories III and IV between the MANCOVA and ANCOVA may be due, in part, to the greater power of MANCOVA, but possibly also implies a multivariate response pattern.

Figure 1 depicts $\mathrm{CBF}$ of the supratentorial cortex and white matter as a function of age for untreated sCS patients, against the background (mean and 95\% CI) generated from control data for brain lobes and the cerebellum. Figure 2 shows the distribution of data for brain lobes and the cerebellum. In controls all four brain lobes and supratentorial cortical matter show an increasing $\mathrm{CBF}$ in the first year of life, with a peak around the age of 4 years, after which there is declining CBF through childhood to an adult level. The cerebellum and supratentorial white matter similarly show a similar age-related pattern with the peak at 1 year of age. On inspection of these graphs it is also evident that the data points from sCS patients do not follow the distribution of data observed in controls. For example, even though all four brain lobes and supratento- rial cortex of sCS patients show a similar developmental progression in $\mathrm{CBF}$ with age to that of controls, they have a lower CBF at the outset of postnatal CBF development and the peak appears to occur at a later age, at approximately 5-6 years. The cerebellum and supratentorial white matter in sCS compared with control subject data also show a similar developmental profile of CBF. The observation of early lower CBF at the outset of postnatal development in sCS patients is supported by significant CBF differences in age category I (Table 3, Fig. 3).

\section{Longitudinal Data}

On general inspection of CBF data in sCS patients (Table 3, Figs. 1-3), it appeared that the CBF of treated sCS patients $>5$ years old was higher than that of untreated sCS patients. Therefore, in a further analysis, we reviewed 12 patients with sCS who underwent serial MRI with ASL before and after vault expansion. The mean age at preoperative MRI was 0.6 years (range $0.2-1.8$ years), and the mean age at postoperative examination was 2.4 years (range 0.9-3.8 years). Figures 4 and 5 summarize the serial ASL measurements in these sCS patients overlaid on normative data by age (mean and $95 \% \mathrm{CI}$ ) in our control subjects. The number of patients whose CBF was below the $95 \%$ CI of control subjects preoperatively versus postoperatively for the frontal lobe changed from $10 / 12$ to $6 / 12$ $(\mathrm{p}=0.125)$, for the parietal lobe from $10 / 12$ to $4 / 12(\mathrm{p}=$ 

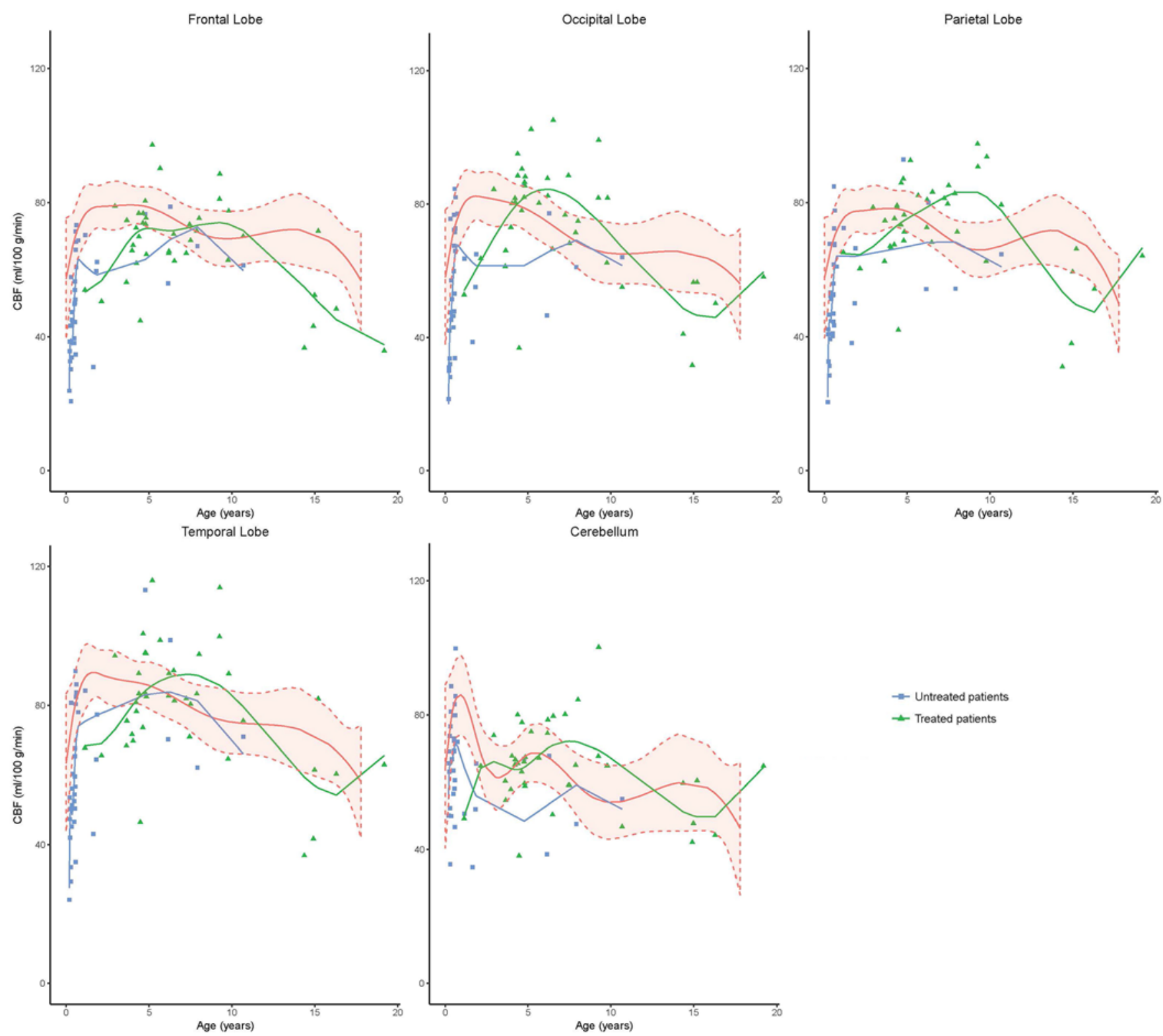

FIG. 2. Distribution of CBF for supratentorial and infratentorial areas in untreated and treated sCS patients by age at measurement, plotted against the distribution of control subject CBF data by age (mean and $95 \% \mathrm{Cl}$ represented by red line and pink shading, respectively). Figure is available in color online only.

$0.070)$, for the occipital lobe from $10 / 12$ to $3 / 12(p=0.065)$, for the temporal lobe from $12 / 12$ to $5 / 12(\mathrm{p}=0.016)$, for the cerebellum from $7 / 12$ to $5 / 12(\mathrm{p}=0.774)$, for the cortical matter from $10 / 12$ to $3 / 12(p=0.065)$, and for white matter from $11 / 12$ to $7 / 12(\mathrm{p}=0.219)$.

\section{Discussion}

In this study of $\mathrm{CBF}$ in children with sCS we have identified three main features when findings are compared with control subject data. First, patients with untreated sCS $<1$ year old have lower than expected CBF. Second, with surgical vault expansion, and age progression, $\mathrm{CBF}$ in sCS patients eventually falls within the range of control subject data. Third, the expected early childhood peak in CBF occurs around the age of 5-6 years in patients with $\mathrm{sCS}$, which is later than that observed in controls.

A key factor in determining the indication and timing of cranial vault expansion in sCS patients is the presence of ICH, or the impending propensity to develop it. Recent discussions have also focused on the way in which abnormal venous anatomy during the first year of life may also impact the development of ICH.11,29 Thus, to better understand the effects of these cranial vault and vascular changes in relation to the postnatal development of CBF in sCS, we sought to use a novel noninvasive MRI technique for assessing CBF, namely ASL. ${ }^{13}$ Previous studies have shown that the ASL-derived estimation of $\mathrm{CBF}$ provides 


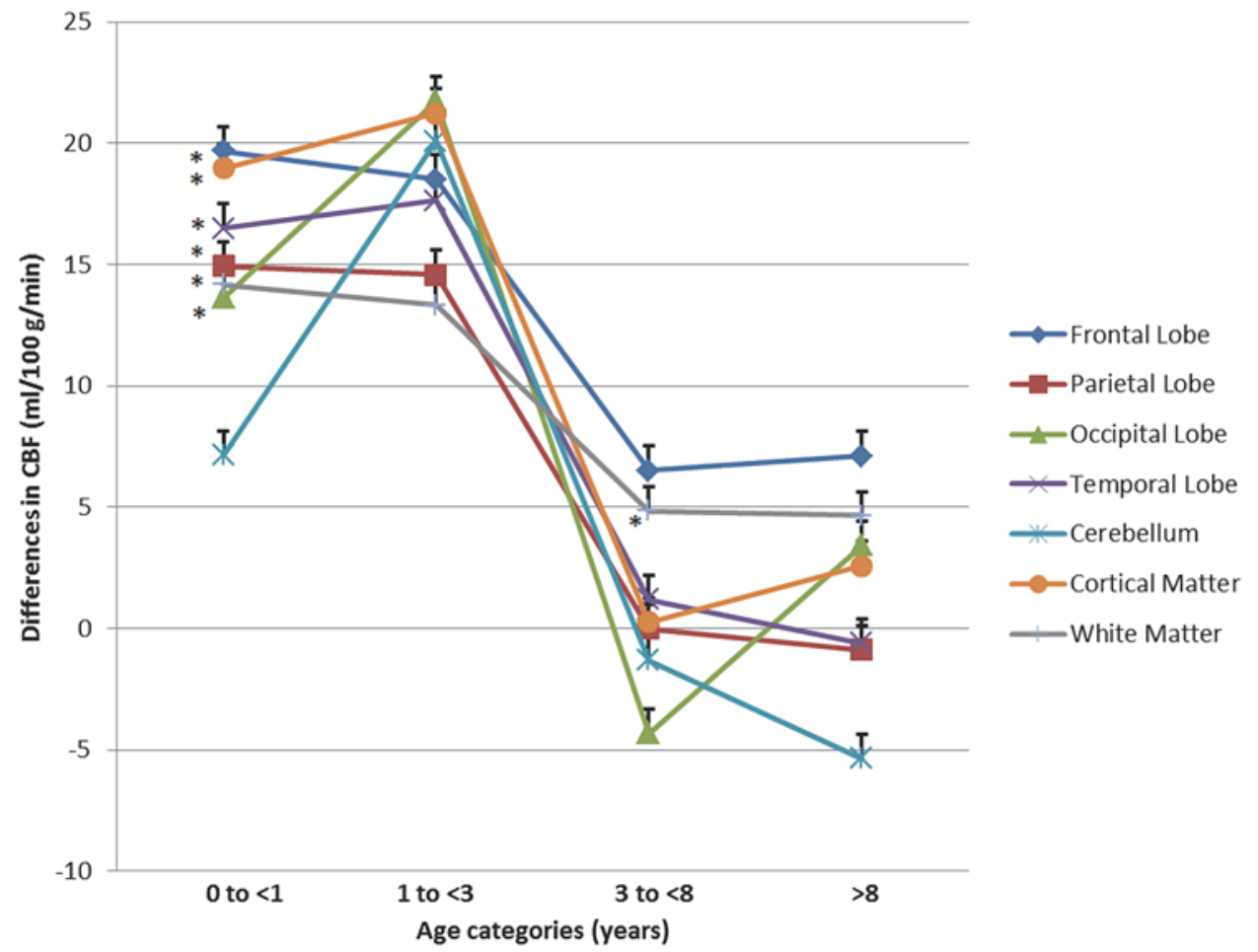

FIG. 3. Mean (and standard error) differences in CBF between control subjects and patients with sCS by age category (years). ${ }^{*}$ Statistical significance in MANCOVA and ANCOVA testing. Figure is available in color online only.

data that are comparable to $\mathrm{CBF}$ values using other techniques, e.g., FDG-PET, SPECT scanning, and dynamic susceptibility contrast MRI. ${ }^{2,16,26} \mathrm{~A}$ recent study by CarsinVu et al. ${ }^{6}$ used ASL to examine CBF in healthy subjects ranging from 0 to 18 years old, and supports the results we found in our controls. That study similarly found an increasing $\mathrm{CBF}$ with a peak at 3-4 years and a decline until adolescence, and found no differences between patients based on sex or anesthesia. One difference was a lower mean CBF in their pediatric population than in ours. Their low CBF values could be the result of using a relatively long PLD of $1800 \mathrm{msec}$, which is recommended in adults. For children a PLD of $1500 \mathrm{msec}$ is recommended, as used in our study. ${ }^{1}$

Previous work using PET scanning in 10 single-suture nonsyndromic craniosynostosis patients showed generalized lower CBF before surgery, which was most pronounced in the frontal lobe and occipital lobe. ${ }^{8}$ We have extended and confirmed these observations with more complete characterization of CBF in 27 patients with sCS in the first year of life. We also describe a generalized reduction in CBF to all brain lobes, especially the frontal lobe. However, the posterior fossa findings were different; we did not observe any differences in cerebellar CBF between untreated sCS patients and controls, which could be due to a homeostatic mechanism that protects the cerebellum. ${ }^{14}$

The potential effect of surgical vault expansion on improving $\mathrm{CBF}$ in $\mathrm{SCS}$ also warrants further discussion. In our study it is difficult to determine whether the apparent normalization of $\mathrm{CBF}$ is due to surgical intervention, or whether it is a natural consequence of age and develop- ment. In the limited series of longitudinal pre- and postoperative CBF measurements, this study could not show statistically significant changes, possibly due to the small number of patients. However, this limited series did show abnormally low CBF before surgery that was higher, and more likely to be within the $95 \%$ CI of controls, after surgery. This finding is of interest because other serial studies focusing on cerebral perfusion ${ }^{4,8,9,24,30}$ and cerebral venous blood flow ${ }^{19}$ in craniosynostosis have also shown improvements after surgical treatment; the interval between preand postoperative measurements varies from 7 days to 4 months in these studies. The only studies on CBF changes with surgery have concentrated on evolution in the tissue underneath the closed suture. ${ }^{4,9,24}$ We now provide global and regional data on CBF before and after surgery.

The pressure-volume curve for brain tissue and the contents of the cranium implies that any increase in brain, blood, or CSF will lead to an increase in intracranial pressure (ICP) once compensatory mechanisms of changes in blood and CSF fail. In sCS, total intracranial volume could be restrictive because the cranial sutures are fused, although this is not prevalent in the first year of life. ${ }^{10,27}$ The impact of increased CSF space/volume observed in our study is not large, as shown by the exploratory findings in SCS patients with hydrocephalus. However, the common finding of elevated ICP in SCS might thus be caused by abnormal venous outflow. Enlarging the skull may potentially normalize CBF not only because it improves blood outflow, but also because it enlarges total intracranial volume, which shifts the pressure-volume curve to the left, reflecting better compliance to shifts in ICP. 

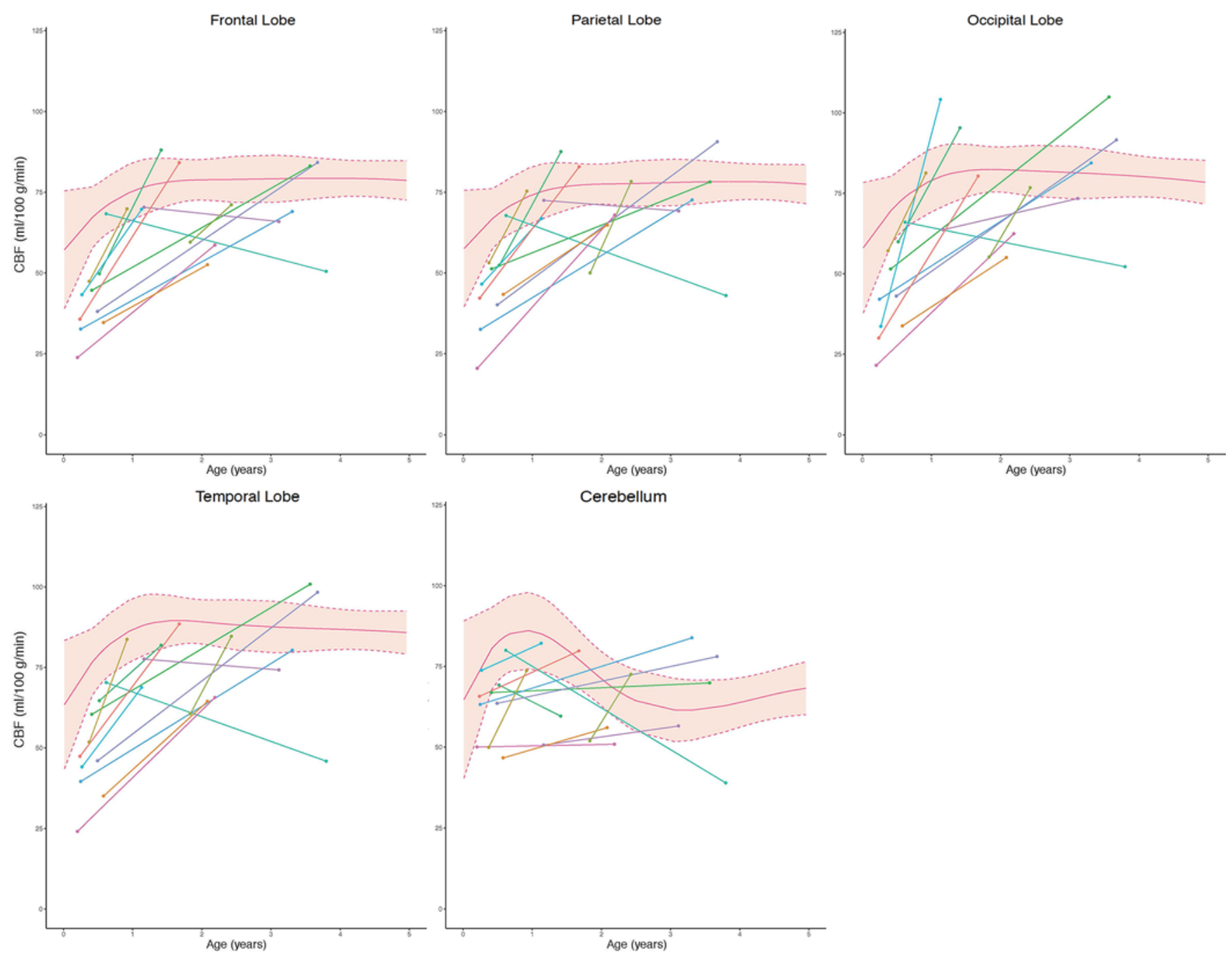

FIG. 4. Longitudinal preoperative to postoperative measurements of CBF in sCS patients against the distribution of control subject $\mathrm{CBF}$ data by age (mean and $95 \% \mathrm{Cl}$ represented by red line and pink shading, respectively). Figure is available in color online only.

After the age of 3 years, $\mathrm{CBF}$ in surgically treated sCS patients appears similar to the level observed in control subjects. However, the distribution in CBF by age is different in children with sCS, with the physiological peak in $\mathrm{CBF}$ occurring at approximately 5-6 years of age. This finding is a new observation and it may offer part of the explanation for the problem of ICH in sCS patients. For example, the age of 5-6 years is the time at which sCS patients often develop $\mathrm{ICH}$, and it is possible that this peak in $\mathrm{CBF}$ is not compensated by abnormal venous anatomy. ${ }^{11}$ We now wonder whether, in such sCS patients, the timing of the peak in a CBF maturational profile is a vulnerable period because of the limit set by outflow, i.e., "outgrowing" drainage capacity.

Our study does have two main limitations, which concern control subject selection and the ASL technique. First, in the matter of control subject selection, age matching would have been our preferred method to obtain control data, but this approach was not possible because of ethical constraints in subjecting healthy young children to the anesthesia required for undergoing MRI examination, solely for research purposes. Hence, we settled for a convenience sample in which a clinical investigation was being performed but there was no evidence of imaging or clinical neuropathology and morbidity, aiming for at least 8 control subjects per age category, based on other cerebral perfusion literature. ${ }^{6,28}$ However, we have been able to use data from nearly 90 control subjects ranging from infants to adults, and while our results in controls are supported by recent literature,${ }^{6}$ we cannot avoid the fact that children included as controls in this study had a reason for undergoing imaging of the head and neck area, and therefore might not be assumed to be perfectly normal. Second, in regard to the ASL measurements and estimation of CBF, there are two limitations that need to be considered. The sensitivity of ASL is reduced in white matter, because CBF is lower and the arterial transit time is longer than that of gray matter. ${ }^{12}$ However, through higher field strength and use of a pseudocontinuous ASL technique, we have addressed this challenge; the narrow 95\% CI for white matter indicates 

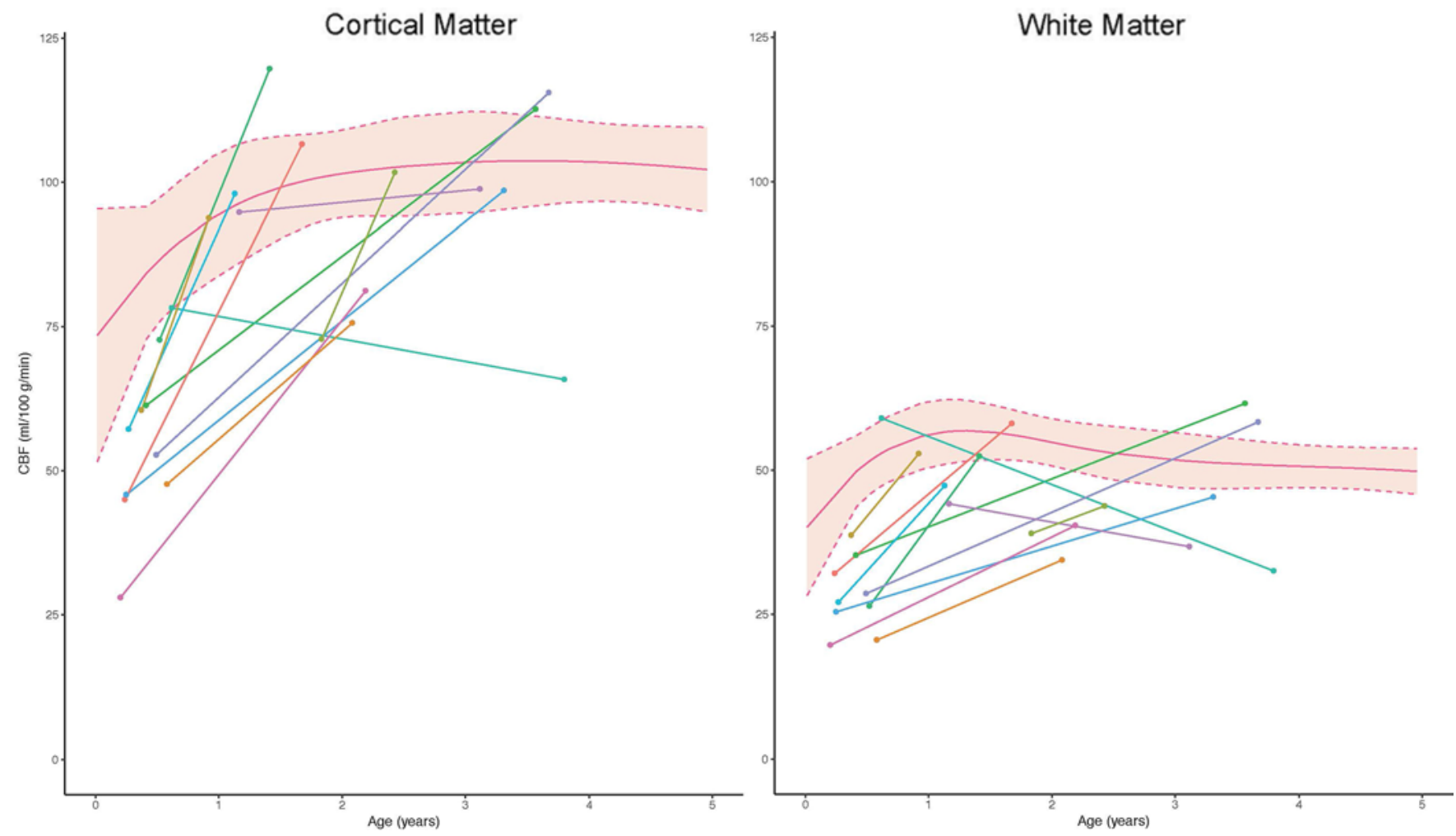

FIG. 5. Longitudinal preoperative to postoperative measurements of CBF (gray matter, white matter) in sCS patients against the distribution of control subject CBF data by age (mean and $95 \% \mathrm{CI}$ represented by red line and pink shading, respectively). Figure is available in color online only.

that our methodology has resulted in an accurate estimate of $\mathrm{CBF}$ in white matter. Next, in regard to our estimation of CBF, the findings may have been affected by a so-called partial volume effect. The partial volume effect relates to the fact that the voxel size in ASL is several times larger than the voxel size available from T1-weighted acquisitions. Hence, most of the voxels measured in ASL contain a combination of tissues and/or CSF, ${ }^{12}$ which may be more pronounced when there is slow development or atrophy of cortical matter. In order to limit the partial volume effect we chose to measure a series of ROIs in three consecutive slices on which the structure of interest was visible, and average the resulting $\mathrm{CBF}$ measurements.

\section{Conclusions}

This case cohort study shows that CBF of untreated children $<1$ year old with sCS is lower than that of controls. After surgical vault expansion in children with $\mathrm{sCS}$, and with age, the expected childhood peak in CBF occurs about 1 year later than that noted in control subjects. After surgery, and with age, children with sCS eventually attain $\mathrm{CBF}$ levels similar to those of control subjects.

\section{Acknowledgments}

We would like to express our very great appreciation to Andrea Poretti for his contribution to creating the ASL measurement protocol, at the Department of Pediatric Radiology of Johns Hopkins University School of Medicine, Baltimore, Maryland.

\section{References}

1. Alsop DC, Detre JA, Golay X, Günther M, Hendrikse J, Hernandez-Garcia L, et al: Recommended implementation of arterial spin-labeled perfusion MRI for clinical applications: A consensus of the ISMRM perfusion study group and the European consortium for ASL in dementia. Magn Reson Med 73:102-116, 2015

2. Armitage PA, Skipper N, Connolly DJ, Griffiths PD: A qualitative comparison of arterial spin labelling and dynamic susceptibility contrast MRI in 52 children with a range of neurological conditions. Br J Radiol 90:20160495, 2017

3. Arnaud E, Meneses P, Lajeunie E, Thorne JA, Marchac D, Renier D: Postoperative mental and morphological outcome for nonsyndromic brachycephaly. Plast Reconstr Surg 110:6-13, 2002

4. Barik M, Bajpai M, Das RR, Malhotra A, Panda SS, Sahoo MK, et al: Role of ${ }^{99 m}$ Tc-ECD SPECT in the management of children with craniosynostosis. Biomed Res Int 2014:172646, 2014

5. Bartels MC, Vaandrager JM, de Jong TH, Simonsz HJ: Visual loss in syndromic craniosynostosis with papilledema but without other symptoms of intracranial hypertension. J Craniofac Surg 15:1019-1024, 2004

6. Carsin-Vu A, Corouge I, Commowick O, Bouzillé G, Barillot C, Ferré JC, et al: Measurement of pediatric regional cerebral blood flow from 6 months to 15 years of age in a clinical population. Eur J Radiol 101:38-44, 2018

7. Cornelissen M, Ottelander Bd, Rizopoulos D, van der Hulst $\mathrm{R}$, Mink van der Molen A, van der Horst C, et al: Increase of prevalence of craniosynostosis. J Craniomaxillofac Surg 44:1273-1279, 2016

8. David LR, Genecov DG, Camastra AA, Wilson JA, Argenta 
LC: Positron emission tomography studies confirm the need for early surgical intervention in patients with single-suture craniosynostosis. J Craniofac Surg 10:38-42, 1999

9. David LR, Wilson JA, Watson NE, Argenta LC: Cerebral perfusion defects secondary to simple craniosynostosis. J Craniofac Surg 7:177-185, 1996

10. de Jong T, Rijken BF, Lequin MH, van Veelen ML, Mathijssen IM: Brain and ventricular volume in patients with syndromic and complex craniosynostosis. Childs Nerv Syst 28:137-140, 2012

11. Florisson JM, Barmpalios G, Lequin M, van Veelen ML, Bannink N, Hayward RD, et al: Venous hypertension in syndromic and complex craniosynostosis: the abnormal anatomy of the jugular foramen and collaterals. J Craniomaxillofac Surg 43:312-318, 2015

12. Grade M, Hernandez Tamames JA, Pizzini FB, Achten E, Golay X, Smits M: A neuroradiologist's guide to arterial spin labeling MRI in clinical practice. Neuroradiology 57:11811202,2015

13. Haller S, Zaharchuk G, Thomas DL, Lovblad KO, Barkhof F, Golay X: Arterial spin labeling perfusion of the brain: emerging clinical applications. Radiology 281:337-356, 2016

14. Huang BY, Castillo M: Hypoxic-ischemic brain injury: imaging findings from birth to adulthood. Radiographics 28:417-439, 617, 2008

15. Johnson D, Wilkie AO: Craniosynostosis. Eur J Hum Genet 19:369-376, 2011

16. Kang KM, Sohn CH, Kim BS, Kim YI, Choi SH, Yun TJ, et al: Correlation of asymmetry indices measured by arterial spin-labeling MR imaging and SPECT in patients with crossed cerebellar diaschisis. AJNR Am J Neuroradiol 36:1662-1668, 2015

17. Maliepaard M, Mathijssen IM, Oosterlaan J, Okkerse JM: Intellectual, behavioral, and emotional functioning in children with syndromic craniosynostosis. Pediatrics 133:e1608 e1615, 2014

18. Mathijssen IM, Arnaud E: Benchmarking for craniosynostosis. J Craniofac Surg 18:436-442, 2007

19. Mursch K, Enk T, Christen HJ, Markakis E, Behnke-Mursch $\mathrm{J}$ : Venous intracranial haemodynamics in children undergoing operative treatment for the repair of craniosynostosis. A prospective study using transcranial colour-coded duplex sonography. Childs Nerv Syst 15:110-118, 1999

20. Renier D, Lajeunie E, Arnaud E, Marchac D: Management of craniosynostoses. Childs Nerv Syst 16:645-658, 2000

21. Renier D, Sainte-Rose C, Marchac D, Hirsch JF: Intracranial pressure in craniostenosis. J Neurosurg 57:370-377, 1982

22. Rijken BF, Leemans A, Lucas Y, van Montfort K, Mathijssen IM, Lequin MH: Diffusion tensor imaging and fiber tractography in children with craniosynostosis syndromes. AJNR Am J Neuroradiol 36:1558-1564, 2015

23. Satoh M, Ishikawa N, Enomoto T, Takeda T, Yoshizawa T, Nose T: [Study by I-123-IMP-SPECT before and after surgery for craniosynostosis.] Kaku Igaku 27:1411-1418, 1990 (Japanese)

24. Sen A, Dougal P, Padhy AK, Bhattacharya A, Kumar R, Bal C, et al: Technetium-99m-HMPAO SPECT cerebral blood flow study in children with craniosynostosis. J Nucl Med 36:394-398, 1995

25. Setia MS: Methodology series module 5: sampling strategies. Indian J Dermatol 61:505-509, 2016
26. Sierra-Marcos A, Carreño M, Setoain X, López-Rueda A, Aparicio J, Donaire A, et al: Accuracy of arterial spin labeling magnetic resonance imaging (MRI) perfusion in detecting the epileptogenic zone in patients with drug-resistant neocortical epilepsy: comparison with electrophysiological data, structural MRI, SISCOM and FDG-PET. Eur J Neurol 23:160-167, 2016

27. Spruijt B, Joosten KF, Driessen C, Rizopoulos D, Naus NC, van der Schroeff MP, et al: Algorithm for the management of intracranial hypertension in children with syndromic craniosynostosis. Plast Reconstr Surg 136:331-340, 2015

28. Takahashi T, Shirane R, Sato S, Yoshimoto T: Developmental changes of cerebral blood flow and oxygen metabolism in children. AJNR Am J Neuroradiol 20:917-922, 1999

29. Tischfield MA, Robson CD, Gilette NM, Chim SM, Sofela FA, DeLisle MM, et al: Cerebral vein malformations result from loss of Twist 1 expression and BMP signaling from skull progenitor cells and dura. Dev Cell 42:445-461.e5, 2017

30. Wang B, Cheng Z, Mu X, Fan B, Guo Z: Preoperative and postoperative transcranial Doppler sonographic evaluations of the cerebral hemodynamics of craniostenosis. J Craniofac Surg 21:432-435, 2010

\section{Disclosures}

Dr. Hernandez-Tamames received support of non-study-related clinical or research effort from GE Healthcare. This study was funded by financial support provided by Stichting Hoofdzaak, a foundation that collects donations for research for patients with craniofacial disorders; they had no involvement in any aspect of the study.

\section{Author Contributions}

Conception and design: Doerga, Lequin, Wagner, Mathijssen. Acquisition of data: Doerga, Lequin, Dremmen, den Ottelander. Analysis and interpretation of data: Doerga, Lequin, Dremmen, Mauff, Wagner, Hernandez-Tamames, Versnel, Joosten, van Veelen, Tasker, Mathijssen. Drafting the article: Doerga. Critically revising the article: all authors. Reviewed submitted version of manuscript: Doerga, Lequin, Dremmen, den Ottelander, Wagner, Hernandez-Tamames, Versnel, Joosten, van Veelen, Tasker, Mathijssen. Approved the final version of the manuscript on behalf of all authors: Doerga. Statistical analysis: Doerga, Mauff, Tasker. Study supervision: Lequin, Mathijssen.

\section{Supplemental Information}

\section{Previous Presentations}

Plenary presentations of this study were given at the 17 th International Congress of the International Society of Craniofacial Surgery (ISCFS) in Cancún, Mexico, on October 17, 2017, and at the European Society of Craniofacial Surgery Biennial Congress in Birmingham, United Kingdom, on September 23, 2016.

\section{Correspondence}

Priya N. Doerga: Sophia Children's Hospital, Dutch Craniofacial Center, Erasmus MC, University Medical Center Rotterdam, The Netherlands.p.doerga@erasmusmc.nl. 